\title{
Ultrafast parametric process in a photonic-crystal nanocavity switch
}

\author{
Lunnemann, Per; Yu, Yi; Joanesarson, Kristoffer; Mørk, Jesper
}

Published in:

Physical Review A

Link to article, DOI:

10.1103/PhysRevA.99.053835

Publication date:

2019

Document Version

Publisher's PDF, also known as Version of record

Link back to DTU Orbit

Citation (APA):

Lunnemann, P., Yu, Y., Joanesarson, K., \& Mørk, J. (2019). Ultrafast parametric process in a photonic-crystal nanocavity switch. Physical Review A, 99(5), [053835]. https://doi.org/10.1103/PhysRevA.99.053835

\section{General rights}

Copyright and moral rights for the publications made accessible in the public portal are retained by the authors and/or other copyright owners and it is a condition of accessing publications that users recognise and abide by the legal requirements associated with these rights.

- Users may download and print one copy of any publication from the public portal for the purpose of private study or research.

- You may not further distribute the material or use it for any profit-making activity or commercial gain

- You may freely distribute the URL identifying the publication in the public portal

If you believe that this document breaches copyright please contact us providing details, and we will remove access to the work immediately and investigate your claim 


\title{
Ultrafast parametric process in a photonic-crystal nanocavity switch
}

\author{
Per Lunnemann, Yi Yu, Kristoffer Joanesarson, and Jesper Mørk ${ }^{*}$ \\ DTU Fotonik, Department of Photonics Engineering, Technical University of Denmark, Ørsteds Plads, DK-2800 Kgs. Lyngby, Denmark
}

(Received 21 December 2018; published 23 May 2019)

\begin{abstract}
We report experimental and theoretical investigations of ultrafast coherent dynamics in a photonic-crystal point-defect nanocavity. The experimental investigations are carried out using a versatile heterodyne pump-probe setup giving access to wavelength and time-resolved dynamics. We find that parametric processes originating from coherent interactions between the pump and the probe lead to characteristic and clearly distinguishable features in the time-resolved dynamics. The parametric process is caused by fast oscillations of spatially trapped carriers, with the nanocavity acting to enhance the optical fields and thereby the strength of these processes. This interpretation is confirmed by the good agreement that is obtained with a theoretical model, which extends previous works by taking into account wave-mixing effects among the probe and pump pulse. The physics behind the parametric process is explained in detail and a simple expression is derived for the conditions under which coherent interactions lead to high-transmission bands for the probe pulses. These results are important for understanding how coherent effects can be used to improve the dynamical properties of nanocavity switches.
\end{abstract}

DOI: 10.1103/PhysRevA.99.053835

\section{INTRODUCTION}

During the past decades, the explosion of data traffic over the Internet has stimulated research on realizing ultrasmall and energy-efficient optical components for photonic integrated circuits [1]. All-optical switches where light is controlled with light are promising for increasing the integration density and bandwidth as well as decreasing the energy consumption in photonic integrated circuits because they offer possibilities for simplifying the circuit structure and reducing electrical interconnects and optoelectronic interfaces [2].

A conceptually simple approach for controlling light with light is to exploit optical nonlinearities, which are, unfortunately, usually extraordinarily weak and, because of that, require long interaction lengths. Photonic-crystal (PhC) nanocavity devices provide a way of enhancing the effective optical nonlinearity by strongly localizing light in an ultrasmall cavity mode volume $(V)$ for an extended period of time, the latter being quantified by the cavity quality factor $(Q$ factor) [3]. With such a combination, the field intensity inside the cavity (scaling as $Q / V$ ) can be significantly enhanced and therefore nonlinear signal processing becomes feasible in ultrasmall devices and at very low pulse energies [4-8].

Conventional $\mathrm{PhC}$ nanocavity switches utilize a strong pump pulse to shift the resonance of a cavity, typically via the dispersion of free carriers excited in the cavity region, and this shift in turn changes the transmission of a probe beam [4-11]. In this case, the switching dynamics is governed by several decay processes characterized by short and long time constants $[6,7,12,13]$. Previous work mainly considered the slow-time-scale dynamics and there is little work on ultrafast coherent dynamics and parametric effects. In terms

\footnotetext{
*jesm@fotonik.dtu.dk
}

of switching, it was shown that coherent excitation of a nanocavity can be used to increase the speed and coupling efficiency [14]. Very recently, using a heterodyne pump-probe technique with subpicosecond temporal resolution [15], we observed effects of coherent wave mixing in the nanocavity as well as the generation of short idler pulses.

Our previous work [15] focused entirely on the wavemixing effects in the time domain. Here, we provide a detailed report on simultaneous wavelength and time-resolved measurements of the ultrafast dynamics in a $\mathrm{PhC}$ nanocavity using optical gating techniques. The measurements show clear signatures of parametric processes, represented by fine spectral "multiband" features appearing in the probe transmission spectrum, which have not been observed before. A theoretical model taking into account wave-mixing effects, which typically are neglected, successfully explains the measurement results. The physics behind the parametric process is explained and an analytic expression is derived for a phase-matching condition, under which the probe pulse can be enhanced by more than $10 \mathrm{~dB}$. The current investigation paves the way for exploiting parametric effects in nanocavities to realize ultrafast switching at low pulse energies.

\section{SETUP AND SAMPLE}

The device investigated is illustrated in Fig. 1(b) and consists of a $\mathrm{PhC}$ membrane structure with input and output line-defect waveguides that are coupled via a point-defect nanocavity. The device geometry is similar to the structures investigated in $[5-7,15]$. If the input probe wavelength matches the resonance frequency of the nanocavity, light can couple from the input waveguide to the output waveguide. Now, by changing the resonance frequency of the nanocavity via a pump signal, the transmission through the device can be optically controlled. The actual device is realized using a 340-nm-thick InP membrane with airhole radius and lattice 


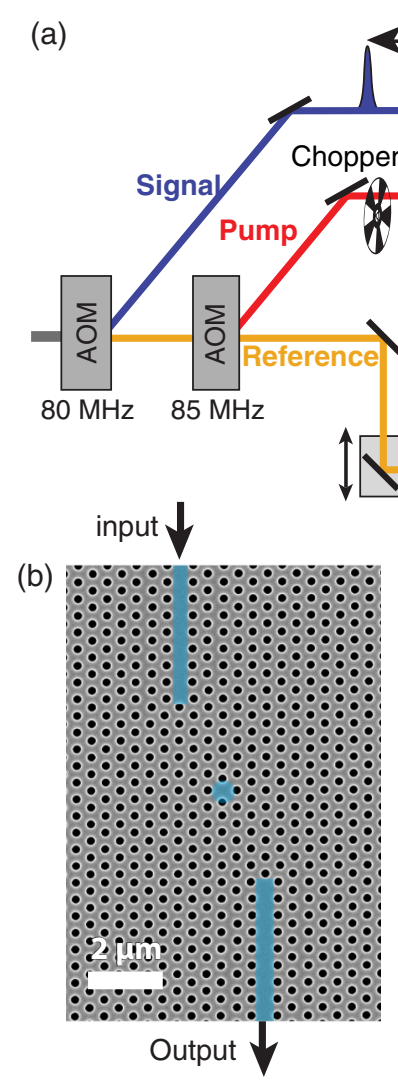

$1 / 79 \mathrm{MHz} 13 \mathrm{~ns}$

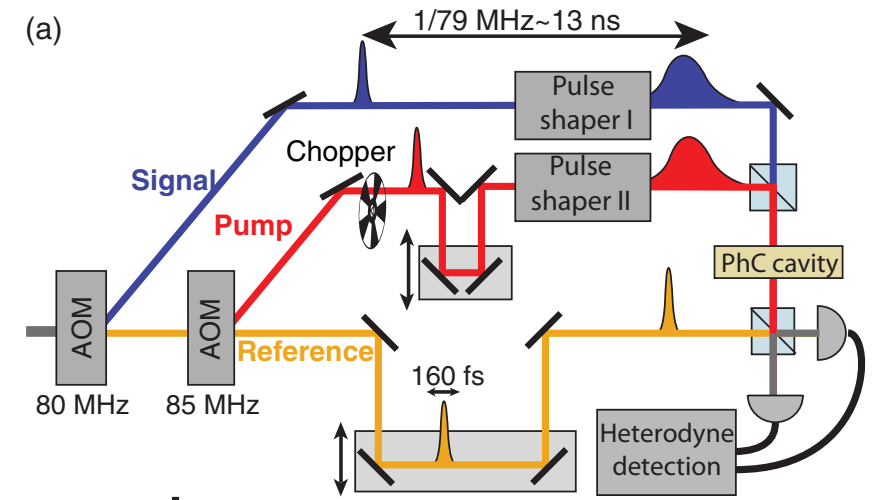

(b)

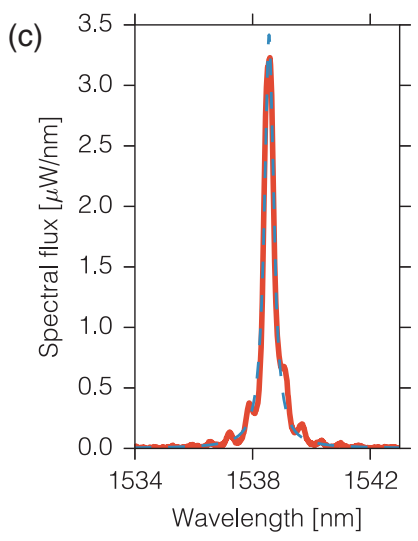

FIG. 1. (a) Sketch of the optical setup. (b) Scanning electron microscope (SEM) image of the sample. Blue coloring serves as a guide for the eye indicating the position of the input and output waveguides and the cavity. (c) Measured transmission spectrum (solid red line) and fitted Lorentzian (dashed blue line) with a spectrum FWHM of $0.37 \mathrm{~nm}$.

constants of 113 and $447 \mathrm{~nm}$, respectively. The cavity was defined by shifting two neighboring holes apart ( $H_{0}$ geometry) with a resonance around $1538.5 \mathrm{~nm}$ and an approximate Lorentzian FWHM of $0.4 \mathrm{~nm}$; cf. Fig. 1(c), corresponding to a total $Q$ factor of $Q=4000$. The intrinsic $Q$ factor of the cavity is $Q_{i} \approx 10^{5}$ as estimated from finite-difference timedomain simulations. The coupling $Q$ factor is $Q_{c}=1 /(1 / Q-$ $\left.1 / Q_{i}\right)$. The cavity is coupled with an in- and output $\mathrm{PhC}$ line-defect waveguide in the fast light regime. The device is equipped with mode adapters [16] to facilitate the out coupling.

The measurements were carried out using a pump-probe setup identical to that in [15] apart from utilizing a different laser. The switching dynamics of the device is investigated using a heterodyne pump-probe technique, where small frequency shifts of the pulses, implemented using acoustooptical modulators, in combination with lock-in techniques are used to separate pump and probe fields $[17,18]$ (see Fig. for a schematic of the setup).

We use a titanium:sapphire pulsed laser (Spectra Physics Maitai) in combination with an optical parametric oscillator (Spectra Physics Inspire HP) providing 160-fs pulses centered around $1.5 \mu \mathrm{m}$ with a repetition rate of $79.9 \mathrm{MHz}$. Two acousto-optical modulators are used for separating the laser
TABLE I. Experimental parameters.

\begin{tabular}{lc}
\hline \hline Parameter & Value \\
\hline Pump spectral width (FWHM) & $1.0 \mathrm{~nm}$ \\
Probe spectral width (FWHM) & $0.5 \mathrm{~nm}$ \\
Incoupled pump pulse energy & $0.1 \mathrm{pJ}$ \\
Incoupled probe pulse energy & $1.0 \mathrm{fJ}$ \\
Estimated incoupling ratio & 0.14 \\
\hline \hline
\end{tabular}

pulses into a train of reference pulses, pump pulses, and probe pulses, being spectrally shifted by 85 and $80 \mathrm{MHz}$ relative to the reference pulse, respectively. A fiber-coupled pulse shaper (Finisar Waveshaper 4000S/X) was used for spectral filtering as well as dispersion compensation. With no spectral filtering, the 160 -fs pulses were distorted and broadened to several picoseconds owing to dispersion in the fiber. By using a feedback-based optimization algorithm for adjusting a thirdorder polynomial phase shift for dispersion compensation, the pulse duration was compressed to well below $200 \mathrm{fs}$. All subsequent spectral filtering for the experiment was implemented with the optimized dispersion compensation. Pump and probe pulses used for the experiment were spectrally filtered using a Gaussian filter (see Table I). With the filters imposed on the pump and the probe being narrow relative to the laser spectrum (FWHM $\sim 25 \mathrm{~nm}$ ), we can assume the spectral components across the filters to be constant, thus carving out a Gaussian spectrum. With the applied dispersion compensation, the filtered pulses were found to be nearly Fourier limited, also showing near-perfect Gaussian pulses in the time domain.

A polarizing beam splitter was used for achieving TE polarization, while quarter- and half-wave plates before the device were used to compensate for the polarization rotation induced by the fibers in the pulse shaper. The transmitted probe or pump pulse was detected using a heterodyne crosscorrelation technique by combining it with the reference pulse on a 50:50 beam splitter. The two outputs were subsequently detected on a fiber-coupled differential detector (Thorlabs PDB450C). The detected signal was sent to a lock-in amplifier (Stanford Research 844) locked to the beating frequency between the laser repetition rate and the modulation frequency of the acousto-optical modulator (10.27 MHz). The pump pulse was modulated using a chopper at a rate of $38 \mathrm{~Hz}$. We note that the short duration of the reference pulse $(\sim 160 \mathrm{fs})$ enables sampling the probe pulse with a high temporal resolution as well as a low detection threshold (a few tens of aJ). We measured a total transmission on resonance of 0.02 , and the pulse energies listed in Table I are estimated energies coupled into the waveguide assuming a symmetric input and output coupling efficiency of $\eta=\sqrt{0.02}$. Important parameters for the pump-probe measurements are listed in Table I.

\section{THEORY}

In order to gain insight into the measured dynamics we make comparisons to a model based on temporal coupled-mode theory as described in $[7,8,19,20]$. However, 
we make the important generalization of including coherent interactions between pump and probe fields, leading to additional interaction and giving rise to the generation of a strong idler field. These equations were derived in the Supplemental Material in Ref. [15], but for clarity and completeness we recapitulate them in the following: We consider the probe to be a small perturbation to the total field in the device, which is dominated by the pump field. This allows us to employ a perturbation expansion of the injected power, the field inside the cavity, and the carrier density. The zeroth-order temporal dynamics describing the transmission of the pump field may then be described by the set of ordinary differential equations $[7,8]$

$$
\begin{aligned}
\frac{d \tilde{a}_{P}}{d t}= & -\left[i \delta_{P}+\kappa_{\mathrm{fc}} N_{1}+\kappa_{\mathrm{tp}}\left|a_{P}\right|^{2}+\gamma\right] \tilde{a}_{P} \\
& +\sqrt{\gamma_{c}} s_{P}(t), \\
\frac{d N_{1}^{(0)}}{d t}= & -\frac{N_{1}^{(0)}-N_{2}^{(0)}}{\tau_{\mathrm{diff}}}-\frac{N_{1}^{(0)}}{\tau_{\mathrm{nr}}}+\frac{\operatorname{Re}\left(\kappa_{\mathrm{tp}}\right)}{\hbar \omega V_{1}}\left|\tilde{a}_{P}\right|^{4}, \\
\frac{d N_{2}^{(0)}}{d t}= & \frac{N_{1}^{(0)}-N_{2}^{(0)}}{\tau_{\mathrm{diff}}} \frac{V_{1}}{V_{2}}-\frac{N_{2}^{(0)}}{\tau_{\mathrm{nr}}},
\end{aligned}
$$

where $\tilde{a}_{P}$ describes the zeroth-order, complex, slowly varying field, $a_{P}(t)=\tilde{a}_{P}(t) e^{-i \omega_{P} t}$, normalized such that $|\tilde{a}|^{2}$ is the energy in the cavity. $s_{P}(t)$ and $s_{p}(t)$ are the amplitude of the incoming pump and probe waves. $N_{1}$ is the carrier density in the region of the nanocavity, the dynamics of which is governed by two-photon absorption (TPA) due to the nanocavity field, fast diffusion to a background density, $N_{2}$, and slower surface and bulk recombination [7]. The volumes $V_{1}$ and $V_{2}$ account for the effective volumes occupied by the different carrier species, $\tau_{\text {diff }}$ is the fast time scale for ambipolar diffusion, and $\tau_{\mathrm{nr}}$ is the longer recombination time. For a detailed discussion of carrier dynamics and diffusion we refer to [7] and [13]. Furthermore, $\delta_{P} \equiv \omega_{0}-\omega_{P}$, where $\omega_{0}$ is the resonance frequency of the cold cavity and $\omega_{P}$ is the frequency of the pump. $\operatorname{Im}\left(\kappa_{\mathrm{fc}}\right)$ denotes free carrier dispersion, $\operatorname{Re}\left(\kappa_{\mathrm{fc}}\right)$ denotes free carrier absorption, $\operatorname{Im}\left(\kappa_{\mathrm{tp}}\right)$ denotes the Kerr effect, and $\operatorname{Re}\left(\kappa_{\mathrm{tp}}\right)$ denotes two-photon absorption. The values of these parameters are given in Refs. [7] and [15]. Here, the spectral shift of the resonance, induced by the pump, is obtained from Eq. (1a) as

$$
\delta_{\mathrm{NL}}(t)=\operatorname{Im}\left(\kappa_{\mathrm{fc}}\right) N_{1}(t)+\operatorname{Im}\left(\kappa_{\mathrm{tp}}\right)\left|\tilde{a}_{P}(t)\right|^{2} .
$$

The associated rate equations for the first-order perturbations, describing the dynamics of the slowly varying weak probe pulse, $\tilde{a}_{p}(t)$, and the generated idler pulse, $\tilde{a}_{i}(t)$, are given as

$$
\begin{aligned}
\frac{\mathrm{d} \tilde{a}_{p}}{\mathrm{~d} t}= & -\left[i \delta_{p} \tilde{a}_{p}+\kappa_{\mathrm{fc}}\left(N_{1}^{(0)} \tilde{a}_{p}+n_{1}^{*} \tilde{a}_{P}\right)\right. \\
& \left.+\kappa_{\mathrm{tp}}\left(2\left|\tilde{a}_{P}\right|^{2} \tilde{a}_{p}+\tilde{a}_{P}^{2} \tilde{a}_{i}^{*}\right)+\gamma \tilde{a}_{p}\right]+\sqrt{\gamma_{c}} s_{p}(t), \\
\frac{\mathrm{d} \tilde{a}_{i}}{\mathrm{~d} t}= & -\left[i \delta_{i} \tilde{a}_{i}+\kappa_{\mathrm{fc}}\left(N_{1}^{(0)} \tilde{a}_{i}+n_{1} \tilde{a}_{P}\right)\right. \\
& \left.+\kappa_{\mathrm{tp}}\left(2\left|\tilde{a}_{P}\right|^{2} \tilde{a}_{i}+\tilde{a}_{P}^{2} \tilde{a}_{p}^{*}\right)+\gamma \tilde{a}_{i}\right],
\end{aligned}
$$

$$
\begin{aligned}
\frac{\mathrm{d} n_{1}}{\mathrm{~d} t}= & i \delta_{P p} n_{1}-\frac{1}{\tau_{\mathrm{diff}}}\left(n_{1}-n_{2}\right)-\frac{1}{\tau_{\mathrm{nr}}} n_{1} \\
& +2 \frac{\operatorname{Re}\left(\kappa_{\mathrm{tp}}\right)}{\hbar \omega V_{1}}\left[\tilde{a}_{P}^{*} \tilde{a}_{i}+\tilde{a}_{P} \tilde{a}_{p}^{*}\right]\left|\tilde{a}_{P}\right|^{2}, \\
\frac{\mathrm{d} n_{2}}{\mathrm{~d} t}= & i \delta_{P p} n_{2}+\frac{1}{\tau_{\mathrm{diff}}}\left(n_{1}-n_{2}\right) \frac{V_{1}}{V_{2}}-\frac{1}{\tau_{\mathrm{nr}}} n_{2},
\end{aligned}
$$

where $\delta_{x}=\omega_{0}-\omega_{x}$, with indices $i, P$, and $p$ denoting idler, pump, and probe, respectively, $\gamma$ is related to the cavity $Q$ factor as $\gamma=\omega_{0} / 2 Q$ and $\gamma_{c}$ is related to the cavity coupling $Q$ factor as $\gamma_{c}=\omega_{0} / 2 Q_{c}$. Also, $\delta_{P p}=\omega_{P}-\omega_{p}$ is the beat frequency between pump and probe at which the slowly varying first-order carrier density amplitudes $n_{1}$ and $n_{2}$ oscillate. Notably, compared to previous models so far used for modeling the switching characteristics $[7,8,13]$, we here include mixing terms such as $\tilde{a}_{P}^{2} a_{i}^{*}$ and $a_{P}^{2} a_{p}^{*}$ in Eqs. (3). The output energy flux is

$$
f_{k} \approx \eta \gamma_{c}\left|\tilde{a}_{k}(t)\right|^{2}, \quad k \in\{i, p, P\} .
$$

The input signals are modeled as Gaussian pulses. The pulse enters the waveguide and propagates down the waveguide before entering the cavity. In our case, the length of the waveguide section is $500 \mu \mathrm{m}$. For the measurements with the highest pump pulse energies, TPA in the entry waveguide is expected to modify the pulse before entering the cavity and we therefore account for TPA in the propagation up to the waveguide

$$
\frac{\mathrm{d} I(z, t)}{\mathrm{d} z}=-\beta_{\mathrm{tp}} I(z, t)^{2},
$$

with the analytic solution

$$
I(z, t)=\frac{I_{\text {in }}(t)}{\beta_{\mathrm{tp}} z I_{\text {in }}(t)+1},
$$

where $I$ is the light intensity, $\beta_{\mathrm{tp}}$ is the TPA coefficient and $I_{\text {in }}(L / 2, t)=\left|s_{P}\right|^{2} / \mathcal{A}$, where $\mathcal{A}$ is an average cross-sectional area of the squared intensity.

\section{EXPERIMENTAL RESULTS}

When coupling in a strong pump pulse prior to the probe, the probe transmission is modified $[8,9]$. Since the band gap in $\operatorname{InP}(1.344 \mathrm{eV})$ is much larger than the excitation wavelength $(\sim 0.80 \mathrm{eV})$, the shift is due to the generation of carriers via two-photon absorption and subsequent free carrier absorption [21] as well as the Kerr effect. The modified transmission of the probe depends both on the mutual delay between the pump and the probe and on the wavelength of the probe. In Fig. 2 the measured change of transmission is presented as a function of the pump-probe delay and probe wavelength for a pump pulse tuned on resonance with the cold cavity and for a fixed probe-reference delay of $0 \mathrm{ps}$.

Considering the normalized transmission [Fig. 2(a)], we note for negative delays (where the pump arrives after the probe) that the transmission is independent of the pump-probe delay. The spectrally resolved transmission is expected to follow a convolution of the cold cavity transmission spectrum (Lorentzian) and probe spectrum (Gaussian) in good agreement with what we observe. For positive pump-probe delay (where the pump arrives before the pulse), we note a distinct band of high transmission that depends both on the 


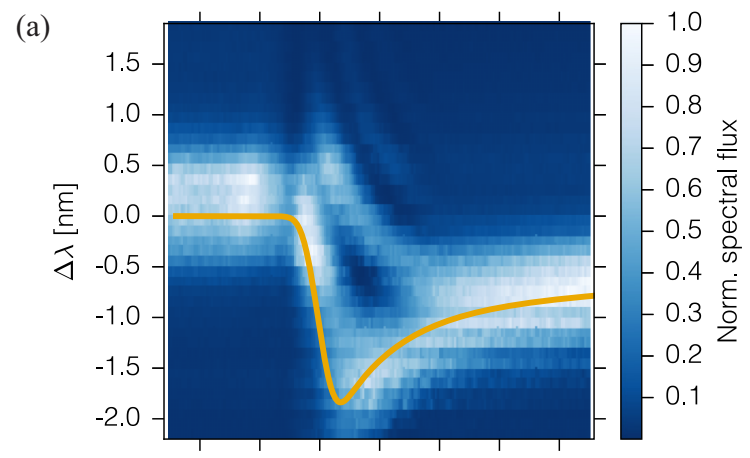

(b)

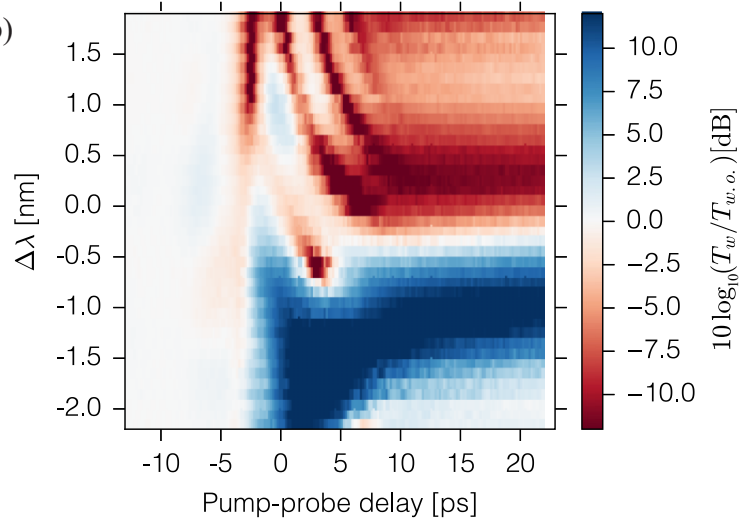

FIG. 2. Measured probe transmission (color scale) versus pumpprobe delay and probe wavelength for a fixed pump wavelength centered at $1538.5 \mathrm{~nm}$. (a) Probe-normalized intensity itself; (b) probe transmission ratio between pump-on and pump-off in logarithmic scale. The orange line in (b) represents the calculated resonance shift.

wavelength and on the pump-probe delay, from which we can clearly resolve the dynamical shift of the resonance. For increasing delays, we note that the transmission window is rapidly blue-shifted up to $1.7 \mathrm{~nm}$ (corresponding to roughly 3.5 times the spectral width of the cold cavity) within a few picoseconds. For delays beyond a few picoseconds, the resonance red-shifts toward its original cold cavity resonance. Considering the measured transmission ratio defined as the ratio of the probe transmission with and without the pump, i.e., $T_{w}$ and $T_{w . o .}$, respectively, presented in Fig. 2(b), we clearly distinguish the wavelengths resulting in a switch-off (switch-on) behavior corresponding to the red (blue) areas with a switching contrast of $\sim 10 \mathrm{~dB}$. For wavelengths slightly blue-shifted (e.g., $-1 \mathrm{~nm}$ ), for increasing delay the probe experiences, first, a transmission enhancement, followed by transmission suppression and, finally, transmission enhancement. In this case, comparing with Fig. 2(a), we immediately see that this oscillating behavior is associated with the resonance being shifted so drastically that it first moves to and subsequently beyond the probe wavelength and, finally, recovers back to the probe wavelength via diffusion and relaxation of the carriers. This directly demonstrates the forth-and-back swing of the resonance in agreement with the conclusions based on single-wavelength pump-probe measurements [9]. It shows that, in contrast to the ordinary linear switching regime, where the cavity is weakly perturbed and the transmitted probe just follows the temporal variation of the cavity resonance, the device operates in a nonlinear regime, where

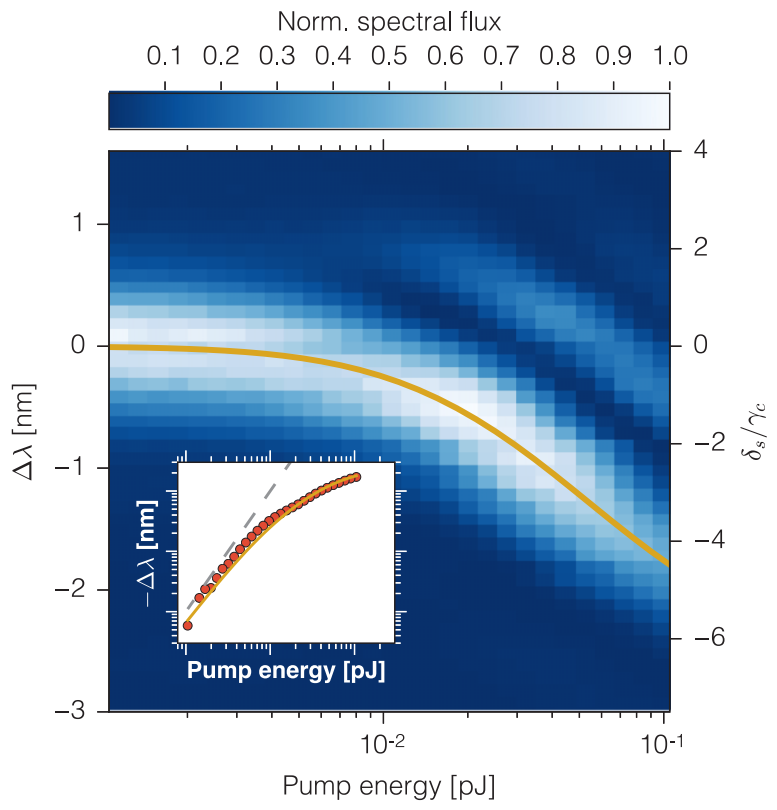

FIG. 3. Measured probe intensity (color scale) versus pump energy and probe wavelength. The pump wavelength was set on resonance with the cavity resonance and the pump-probe delay is fixed at $5 \mathrm{ps}$. The solid orange line represents the theoretically calculated mean wavelength of the cavity resonance, based on coupled-mode theory. Inset: Same plot on a double-log axis. Red circles show the calculated mean wavelength based on a Gaussian fit. The solid gray line is a guide for the eye considering a wavelength shift that scales as the pump energy squared.

the cavity is strongly perturbed by the pump pulses, leading to the initial reduction of the probe transmission. Finally, we note from the differential transmission in Fig. 2(b), distinct fast oscillations as a function of the pump delay, for the probe being red-shifted relative to the cold cavity resonance. Inspecting the transmission data [Fig. 2(a)], this appears as a second and (by close inspection) a third resonance with a transmission enhancement of $10 \mathrm{~dB}$. We return to these oscillations at the end of the section.

The spectral shift induced by the pump pulse naturally depends on its energy. Typically, the spectral shift is recorded by measuring the transmitted spectrum of a spectrally broad pump pulse [8]. This type of measurement often gives rise to a double-peaked spectrum for large input powers since the resonance shift is dynamically shifted, thus containing features of both the cold and the perturbed resonance. The exact spectral profile of the perturbed resonance is therefore difficult to resolve, which is of importance for the switching performance. To this end we carried out a gated spectral measurement by setting the pump at a fixed positive delay, relative to the probe, and subsequently measuring the transmission of the probe. The delay was chosen to be $5 \mathrm{ps}$, serving as a good compromise between having a large spectral shift of the resonance [see Fig. 2(a)], while the spatial overlap between the pump and probe (outside the device) is limited. The results are presented in Fig. 3.

We note that the resonance is monotonically blue-shifted for increasing pump power. Inspecting the resonance shift as a function of the pump energy on a double-logarithmic plot, 


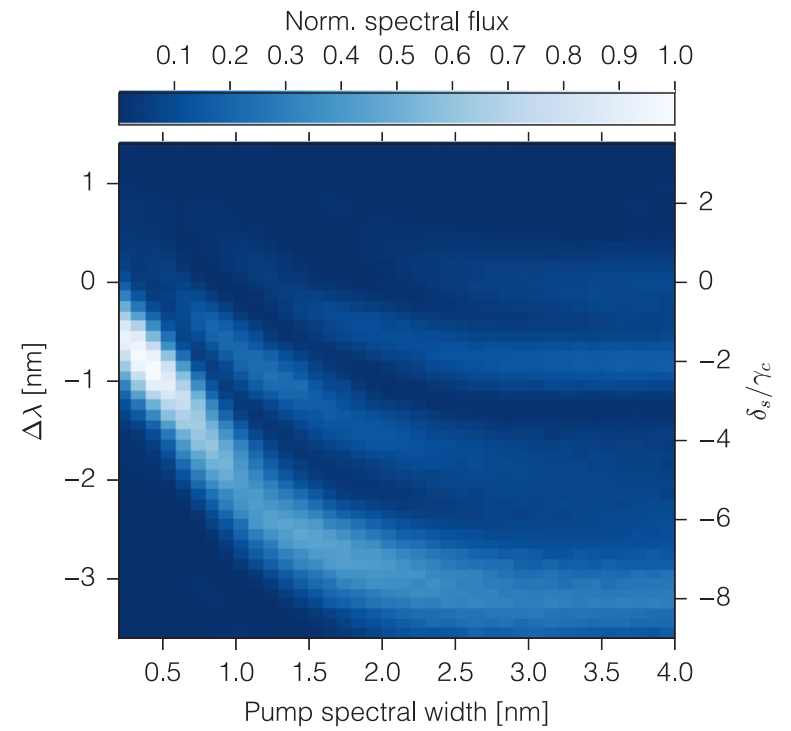

FIG. 4. Measured probe intensity (color scale) versus pump spectral width (FWHM) and probe wavelength. The pump pulse energy is fixed at $0.15 \mathrm{pJ}$ and the pump wavelength is set on resonance with the cavity. Furthermore, the pump-probe delay is fixed at 5 ps.

we clearly see that for the lowest pump energies, where the density of free carriers generated by TPA also is low, the resonance shift scales with the pump energy squared, i.e., $\delta_{\mathrm{NL}}=\operatorname{Im}\left(\kappa_{\mathrm{fc}}\right) \frac{\operatorname{Re}\left(\kappa_{\mathrm{tp}}\right)}{\hbar \omega V_{1}}\left|\tilde{a}_{0}\right|^{4} \Delta \tau$, considering Eq. (2) in combination with (1b) in the limit of $\left(N_{1}^{(0)}-N_{2}^{(0)}\right) / \tau_{\text {diff }} \rightarrow 0$ and $N_{1}^{(0)} / \tau_{\mathrm{nr}} \rightarrow 0$, with $\Delta \tau$ being the pulse width. The results are in very good agreement with the calculated resonance shift found from Eq. (2). From the calculations we find that setting $\operatorname{Im}\left(\kappa_{\mathrm{tp}}\right)=0$ did not significantly change the calculated resonance shift, thus leading us to conclude that for the current experimental parameters only the free carrier dispersion is responsible for the spectral shift. Importantly, for pump energies $>0.01 \mathrm{pJ}$ we note additional spectral features that are red-shifted relative to the main resonance, which are not captured by the expression for the resonance shift, Eq. (2). We return to the origin of this in the next section. While the experimental data do not show clear signs of saturation of the resonance shift, we found from calculations that beyond $0.1 \mathrm{pJ}$ the spectral shift saturates. For such high input energies, the cavity resonance is significantly spectrally shifted during the initial part of the pump pulse, thus inhibiting the remaining tail of the pump pulse to couple to the cavity. Naturally, this can be compensated for by increasing the spectral width of the pump pulse by using a broader spectral filter, at the expense of lowering the coupling efficiency. It was shown in [14] and [22] that by chirping the input pulse the coupling to the cavity can be increased.

To confirm this we carried out measurements, similar to those in Fig. 3 but keeping the pump pulse energy fixed at $0.15 \mathrm{pJ}$ while varying the spectral width of the pump. The results are presented in Fig. 4. In the figure we clearly observe that the shift of the resonance increases with increasing spectral width of the pump until it reaches $2 \mathrm{~nm}$. This reflects a competition between two effects: a broader pump

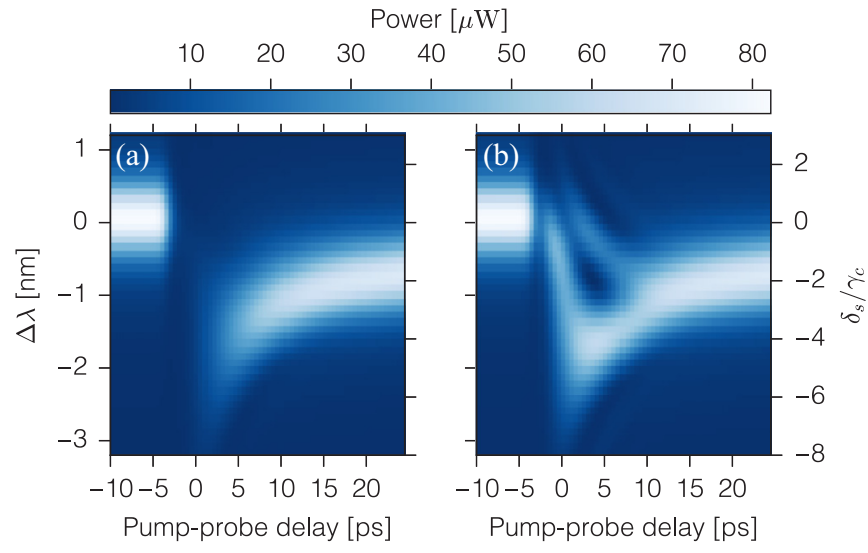

FIG. 5. Calculated probe intensity versus pump-probe delay and probe wavelength (a) without and (b) including the effects of coherent mixing. The pump pulse energy is fixed at $0.1 \mathrm{pJ}$

spectral width facilitates continuous light coupling into the cavity during switching but, at the same time, leads to an overall decrease in the coupling efficiency, thus decreasing the generation rate of free carriers. Finally, we note that, similarly to Figs. 2 and 3, we observe several spectral bands of higher transmission. Thus, for a pump spectral width of $3 \mathrm{~nm}$ we may resolve up to three additional resonance resonance bands that are red-shifted relative to the primary resonance. In the following we address these spectral features.

\section{ANALYSIS OF SPECTRAL FEATURES}

In order to separate the effect of coherent interactions, we carried out calculations with and without the coherent mixing terms. In the former case we solved Eq. (3) and in the latter we used the model presented in [7], corresponding to neglecting all four-wave mixing terms.

In Fig. 5 we present the results of the calculated probe transmission as a function of the pump-probe delay and probe spectral detuning, similar to the experimental results in Fig. 2. Figure 5(a) shows results without coherent interactions taken into account, while Fig. 5(b) shows results including coherent interactions. While Fig. 5(a) clearly demonstrates a spectral shift of the cavity resonance at the arrival time of the pump, we only resolve a single resonance. On the other hand, when coherent interactions are included [Fig. 5(b)], we note the appearance of additional "bands" of high transmission for positive pump-probe delays, in good qualitative agreement with the measurements in Fig. 2.

In Fig. 6 we show the calculated probe transmission as a function of the pump energy and probe spectral detuning, similar to the experimental results in Fig. 3, for the two cases of omitting [Fig. 6(a)] and including [Fig. 6(b)] coherent interactions. The pump-probe delay is fixed at $5 \mathrm{ps.} \mathrm{We} \mathrm{observe}$ that good qualitative agreement with the measurements in Fig. 4 is only observed upon including coherent interactions, which lead to a second and (faint) third resonance that are blue-shifted relative to the primary resonance.

Next we discuss the physical origin of the additional resonances (high-transmission bands) that appear due to coherent interactions. Consider the theoretical model in which we 


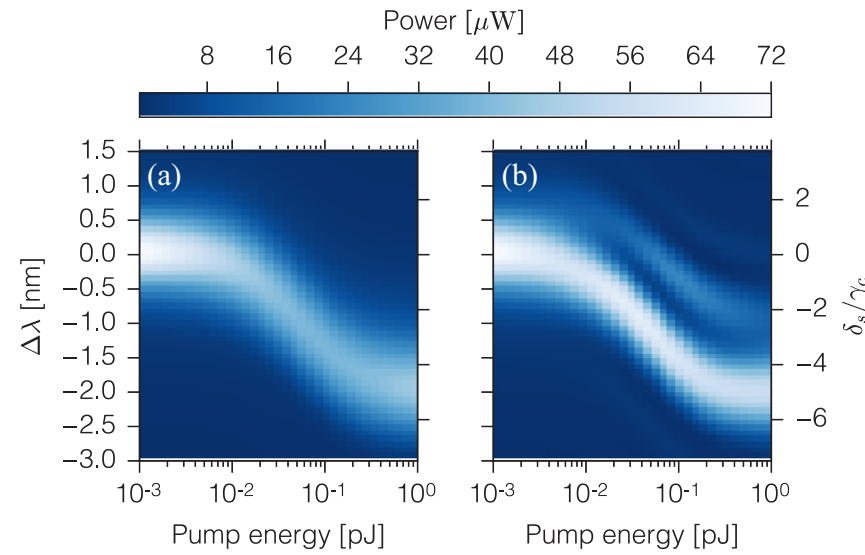

FIG. 6. Calculated spectral shift as a function of the pump pulse energy (a) without and (b) including coherent effects. The pumpprobe delay was fixed at $5 \mathrm{ps}$.

separate the amplitude and phase of the fields $\tilde{a}_{k}=\left|\tilde{a}_{k}\right| e^{j \phi_{k}}$ and $n_{1}=\left|n_{1}\right| e^{j \phi_{n}}$ in Eq. (3). For simplicity, we neglect the idler $\tilde{a}_{i}$ and the slow carrier density component $n_{2}$ as well as nonlinear losses. Since we previously found that the Kerr effect is orders of magnitude smaller than the free carrier effect [15] we may safely also neglect this in the following. This leads to the following simplified equation for the probe field:

$$
\begin{aligned}
\frac{d\left|\tilde{a}_{p}(t)\right|}{d t}= & \operatorname{Re}\left(\kappa_{\mathrm{fc}}\right)\left|n_{1}(t) \tilde{a}_{P}(t)\right| \\
& \times \sin \left(\phi_{P}(t)-\phi_{n}(t)-\phi_{p}(t)\right) \\
& +-\gamma\left|\tilde{a}_{p}(t)\right|+\sqrt{\gamma_{c}} s_{p}(t) \cos \left(\phi_{p}(t)\right) .
\end{aligned}
$$

As can be seen, $\Delta \phi(t)=\phi_{P}(t)-\phi_{p}(t)-\phi_{n}(t)$ represents a phase mismatch, with $\Delta \phi=2 n \pi+\pi / 2$ representing complete phase matching, where the adiabatically shifted pump field is efficiently scattered into the probe field via fourwave mixing. Unlike wave mixing mediated by instantaneous nonlinearities, such as the Kerr effect, the phase mismatch depends on the pump and probe phase, as well as the phase of the oscillating carrier density, which represents a temporal grating. The phases of the fields $\phi_{k}(t)$ are typically correlated with each other through their mutual interaction. However, in the simulations we found this effect to be relatively small, allowing us to represent the phase variations as $\phi_{P}(t) \simeq \theta_{P}(\tau)-$ $\delta_{\mathrm{NL}}(\tau)(t-\tau)$ and $\phi_{n}(t) \simeq \delta_{p}(t-\tau)$ (as the pump frequency is set at the cavity resonance frequency so $\delta_{n}=\delta_{p}$ ). Here, $\tau$ denotes the pump-probe delay, and $\theta_{P}(\tau)$ and $\delta_{\mathrm{NL}}(\tau)$ denote the phase of $\tilde{a}_{P}$ and the blue shift of the cavity resonance, respectively. This can also be understood physically: as the pump pulse enters the cavity, the resonance is blue-shifted and the intracavity field is adiabatically shifted by the same amount $[23,24]$. To derive these, we have assumed that the pump wavelength coincides with the resonance peak, as in our experimental situation, and we have also made the assumption that the carrier densities do not change much, considering that the carrier lifetime is much longer than the probe pulse width. $\phi_{p}(t)$ is the cavity-induced phase change between $\tilde{a}_{p}$ and $s_{p}$ and depends on the probe detuning. In the steady state, the phase change $\phi_{p}(t)$ follows an error-function-like function

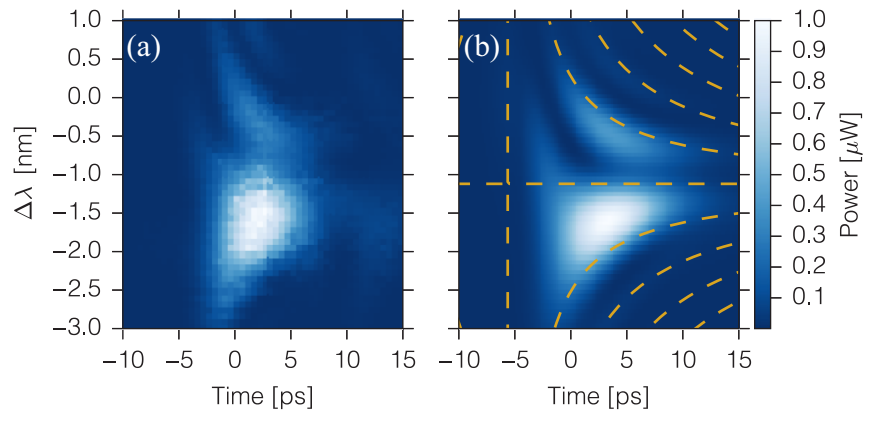

FIG. 7. (a) Measured and (b) simulated probe intensity (color scale) versus time and probe detuning. Dashed yellow lines in (b) indicate values fulfilling the condition $\sin \left(\varphi-\left(\delta_{p}+\delta_{\mathrm{NL}}(\tau)\right)(t+\right.$ $\left.\left.t_{0}\right)\right)=1$, for $\varphi=\pi / 2, \delta_{\mathrm{NL}}(\tau)=5.6 \gamma, \tau=5 \mathrm{ps}$, and $t_{0}=5.5 \mathrm{ps}$.

varying between $-\pi / 2$ and $\pi / 2$ across the resonance and approaches a constant of $-\pi / 2(\pi / 2)$ on the red (blue) side (by using the exponential factor $-j \omega t$ ) of the resonance for large detuning. In our case, the probe spectral width is more than two times narrower than the cavity spectral width; this means that the phase change of the probe approaches the value of its steady state but with some attenuation, i.e., the slope of the phase variation with frequency becomes smaller and the phase change does not become entirely $\pm \pi / 2$ when the probe is far detuned, so we can approximate the phase change by a first-order expansion around $\delta_{p}=0$, i.e., we treat the variation of $\phi_{p}(t)$ as a time-independent variable, approximating it as a linear function of $\delta_{p}: \phi_{p}\left(\delta_{p}\right)=t_{0} \delta_{p}$ with an expansion coefficient $t_{0}$ which is a constant. As a result, in the case where the probe detuning $\phi_{p}$ is varied but the other parameters are fixed, we may describe the phase mismatch in a very simple form as

$$
\Delta \phi(t)=-\left(\delta_{p}+\delta_{\mathrm{NL}}(\tau)\right)\left(t+t_{0}\right)+\varphi,
$$

where $\varphi=t_{0} \delta_{\mathrm{NL}}(\tau)+\theta_{P}(\tau)$. Here we have rescaled the time $t$ so that $t=0$ corresponds to the time when the probe pulse enters the cavity. Equation (7) indicates that $1 /\left(\delta_{p}+\delta_{\mathrm{NL}}\right)$ governs the "period" of the spectral "bands" where the phasematching condition is fulfilled and the transmitted probe field is enhanced. At fist glance, the "period" may look similar to that of the beating effect between the pump and the probe fields, which can also give rise to small oscillations on the probe pulse envelope [7]. However, in the present case the pump energy is scattered to the spectral location of the probe, meaning the probe field intensity is strongly enhanced at the oscillation peaks of the probe pulse envelope. In contrast, there is no enhancement of the probe pulse energy in the simpler beating case considered in [7].

To confirm this interpretation, we carried out measurements of the transmitted probe envelope for a range of probe detunings, $\delta_{p}$. Our heterodyne pump-probe setup resolution allows measurement of the probe pulse shape directly with subpicosecond resolution [15]. The measured result is shown in Fig. 7(a) and agrees well with the corresponding theoretically calculated result in Fig. 7(b). In both cases, spectral bands representing a $>10-\mathrm{dB}$ enhancement of the probe pulse intensity are observed. We have also performed calculations where the coherent interactions are neglected, in which case 
the spectral "multiband" features disappear. In Fig. 7(b), the dashed yellow lines show the contour lines where the function $\sin \left(\varphi-\left(\delta_{p}+\delta_{\mathrm{NL}}(\tau)\right)\left(t+t_{0}\right)\right)$ assumes its maximum value of 1 . The overlap with the high-transmission spectral bands confirms our interpretation of the additional resonances as being due to phase matching.

\section{CONCLUSION}

In conclusion, we have investigated optical switching in a nonlinear $\mathrm{PhC}$ nanocavity switch using gated measurements based on a heterodyne pump-probe setup. A nonlinear switching regime where the cavity is strongly perturbed by the pump pulses was observed, leading to an initial reduction of the probe transmission, similar to previous observations [9]. In addition, the measurements show unusual spectral "multiband" features which have not been observed before. By comparing the measurements to a model based on coupled mode theory and including four wave-mixing effects, we were able to interpret the results as resulting from enhanced light transmission due to parametric gain. As opposed to nonlinear mixing due to Kerr effects [25], the parametric gain is induced by cavity-enhanced oscillations of carriers in a local region around the nanocavity. Based on the theoretical model, we have discussed the detailed physical picture and developed a simple equation governing the phase-matching condition for the probe. Compared to conventional nanocavity-based optical switches [6,11], which showed limitations in terms of switching speed due to the slow component of the carrier dynamics brought about by the long carrier lifetime, the parametric effects bring the advantages of faster optical switching in combination with low energy consumption because it is the fast carrier diffusion from the nanocavity that suppresses the beating between the pump and the probe, which is at the origin of the parametric process.

\section{ACKNOWLEDGMENTS}

The authors acknowledge financial support from Villum Fonden via the NATEC (Nanophotonics for Terabit Communications) Centre (Grant No. 8692) as well as the Danish Research Council for Independent Research (Grant No. DFF - 4181-00416).
[1] D. A. Miller, J. Light. Technol. 35, 346 (2017).

[2] R. Ho, K. W. Mai, and M. A. Horowitz, Proc. IEEE 89, 490 (2001).

[3] M. Notomi, A. Shinya, K. Nozaki, T. Tanabe, S. Matsuo, E. Kuramochi, T. Sato, H. Taniyama, and H. Sumikura, IET Circuits, Devices Syst. 5, 84 (2011).

[4] T. Tanabe, M. Notomi, S. Mitsugi, A. Shinya, and E. Kuramochi, Opt. Lett. 30, 2575 (2005).

[5] C. Husko, A. De Rossi, S. Combrié, Q. V. Tran, F. Raineri, and C. W. Wong, Appl. Phys. Lett. 94, 021111 (2009).

[6] K. Nozaki, T. Tanabe, A. Shinya, S. Matsuo, T. Sato, H. Taniyama, and M. Notomi, Nat. Photon. 4, 477 (2010).

[7] Y. Yu, E. Palushani, M. Heuck, N. Kuznetsova, P. T. Kristensen, S. Ek, D. Vukovic, C. Peucheret, L. K. Oxenlowe, S. Combrié, A. de Rossi, K. Yvind, and J. Mork, Opt. Express 21, 31047 (2013).

[8] M. Heuck, S. Combrié, G. Lehoucq, S. Malaguti, G. Bellanca, S. Trillo, P. T. Kristensen, J. Mørk, J. Reithmaier, and A. De Rossi, Appl. Phys. Lett. 103, 181120 (2013).

[9] Y. Yu, E. Palushani, M. Heuck, D. Vukovic, C. Peucheret, K. Yvind, and J. Mork, Appl. Phys. Lett. 105, 071112 (2014).

[10] Y. Yu, M. Heuck, H. Hu, W. Xue, C. Peucheret, Y. Chen, L. K. Oxenløwe, K. Yvind, and J. Mørk, Appl. Phys. Lett. 105, 061117 (2014).

[11] Y. Yu, H. Hu, L. K. Oxenløwe, K. Yvind, and J. Mork, Opt. Lett. 40, 2357 (2015).
[12] T. Tanabe, H. Taniyama, and M. Notomi, J. Lightwave Technol. 26, 1396 (2008).

[13] G. Moille, S. Combrié, and A. De Rossi, Phys. Rev. A 94, 023814 (2016).

[14] P. Trøst Kristensen, M. Heuck, and J. Mørk, Appl. Phys. Lett. 102, 041107 (2013).

[15] P. Colman, P. Lunnemann, Y. Yu, and J. Mørk, Phys. Rev. Lett. 117, 233901 (2016).

[16] Q. V. Tran, S. Combrié, P. Colman, and A. De Rossi, Appl. Phys. Lett. 95, 061105 (2009).

[17] A. Mecozzi and J. Mørk, JOSA B 13, 2437 (1996).

[18] P. Borri, J. Mørk, and J. M. Hvam, Opt. Commun. 169, 317 (1999).

[19] A. de Rossi, M. Lauritano, S. Combrié, Q. V. Tran, and C. Husko, Phys. Rev. A 79, 043818 (2009).

[20] P. T. Kristensen, J. R. de Lasson, M. Heuck, N. Gregersen, and J. Mork, J. Light. Technol. 35, 4247 (2017).

[21] P. Lunnemann, S. Ek, K. Yvind, R. Piron, and J. Mørk, New J. Phys. 14, 013042 (2012).

[22] S. Serna, J. Oden, M. Hanna, C. Caer, X. L. Roux, C. Sauvan, P. Delaye, E. Cassan, and N. Dubreuil, Opt. Express 23, 29964 (2015).

[23] D. M. Beggs, I. H. Rey, T. Kampfrath, N. Rotenberg, L. Kuipers, and T. F. Krauss, Phys. Rev. Lett. 108, 213901 (2012).

[24] M. Notomi, Rep. Prog. Phys. 73, 96501 (2010).

[25] C. Monat, M. Ebnali-Heidari, C. Grillet, B. Corcoran, B. J. Eggleton, T. P. White, L. O'Faolain, J. Li, and T. F. Krauss, Opt. Express 18, 22915 (2010). 